\title{
Art and the machine
}

You see them every day: smartphone-surfing pedestrians, coffee shops full of transfixed laptop tappers and train carriages replete with tablet gawkers. The computer has certainly evolved with time, not only with regards to function, but also aesthetically - coming in all shapes, sizes and colours. This often sleek, lightweight technology, which can now process data at a speed once considered science fiction, has become the ultimate fashion accessory. But how did this happen?

The exhibition Thinking Machines: Art and Design in the Computer Age at the Museum of Modern Art in New York is a reflection on the collaboration between art and computers from 1959 to 1989. At a time when the computer's function was rather mystical except to scientists and engineers, artists worked alongside this 'emerging technology' by partnering with institutions and corporations in order to explore the production of art. The exhibition is a mixture of installations, drawings, prints and film, as well as computer models from the era. It features works by artists such as Beryl Korot, Vera Molnár, Cedric Price and Stan VanDerBeek, with computers designed by Thinking Machines Corporation, IBM, Olivetti and Apple. The result is a journey through time - exploring the function and aesthetics of computers, but also their purpose in the working environment and the effect they have on social interaction.

The exhibition presents the computer as machine, medium and method for art. For example, the sheet music of HPSCHD by composers John Cage and Lejaren Hiller is displayed, which is a collection of compositions for harpsichord. The music was produced by taking randomly processed pieces by famous classical composers and using Fortran to create computer-generated tapes of the compositions.

Computer-generated art has also been political in nature, as in Waldemar Cordeiro's piece, Gente Ampli². Working with Giorgio Moscati, a physicist at the University of São Paulo, Cordeiro digitized a photograph of a demonstration against military dictatorship and printed the result using alphanumeric characters.

Also on display is the CM-2 supercomputer, produced by artist and

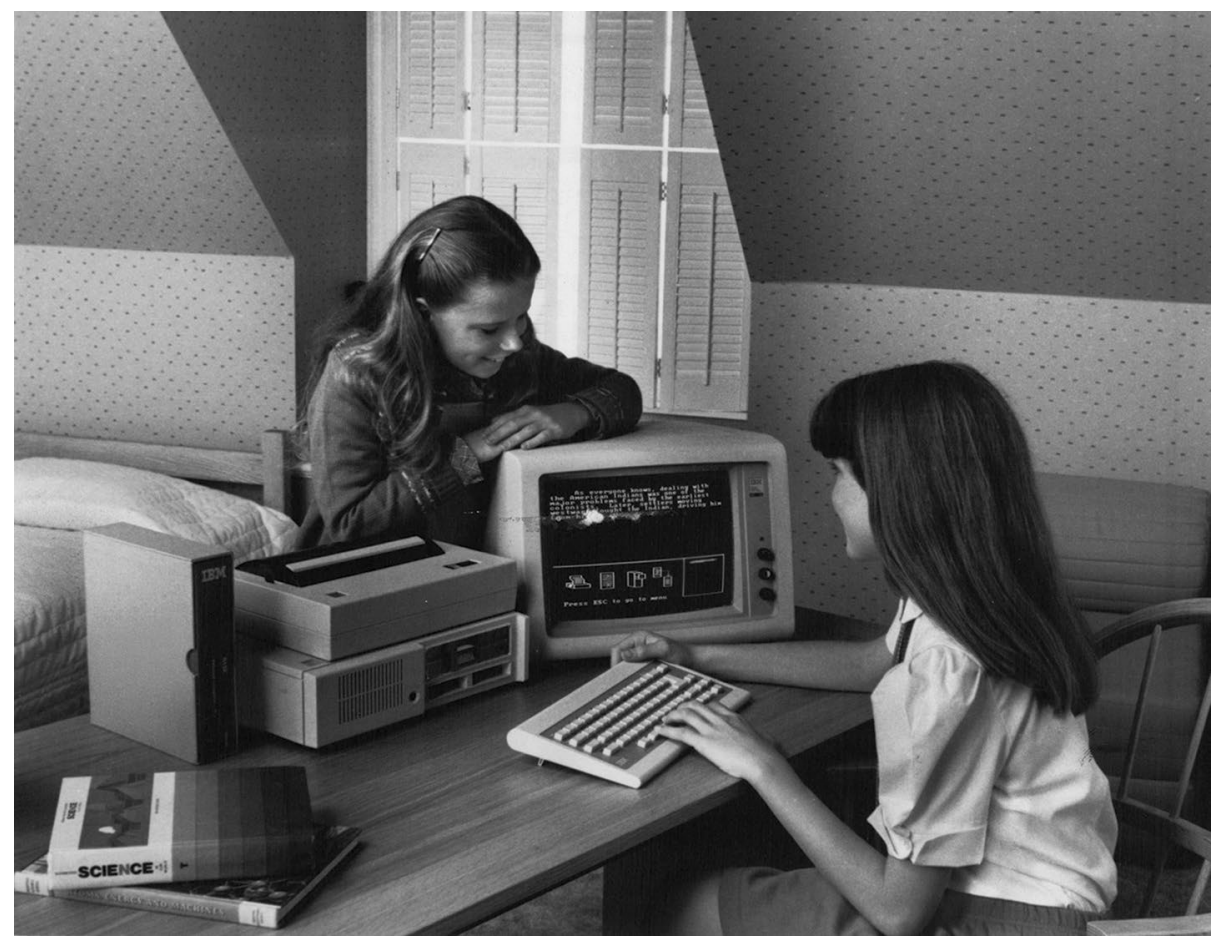

Credit: ZUMA Press, Inc. / Alamy Stock Photo

designer Tamiko Thiel for the Thinking Machines Corporation, which was one of the first commercial supercomputers designed for applications in artificial intelligence. With $512 \mathrm{MB}$ of RAM, a hard drive capacity of $25 \mathrm{~GB}$, and standing $1.5 \mathrm{~m}$ tall, it is a vivid reminder of the progress of technology. Amusingly, the designer increased the number of LEDs on the exterior of the machine in order to increase its decorative effect.

More interesting was the period in the 1980s that saw the proliferation of computers in daily life and the work environment. The Massachusetts Institute of Technology invited artist Lee Friedlander to make a series of photographs depicting office workers gazing hypnotically at their computer screens. The implication that our daily routine is so interlinked with these machines that they become a reflection of one's self is at once scary and thoughtprovoking. Perhaps it's time to clear up that desktop junk!

For the technologically nostalgic, the exhibition also displays some of the first personal computers that entered the market, including the first Macintosh XL (1985) and a DIAB DS-101 Computer (1985-1989), which was designed by British artist Richard Hamilton.

The driving force for these artists to work with computers was really experimental exploration; they wanted to understand what could be done with them and how they would impact society. Looking back, you can't but appreciate how timely and important this was. The exhibition does very well to showcase the development of this collaboration from the 1960s through to the 1980s. It is quite clear - perhaps unsurprisingly - that artists and designers had a lot to do with the demystification of the black box computer, making it more accessible, usable and aesthetically appealing to the general public. You might hope that artists ride the wave of every emerging technology that is yet to come.

\section{Reviewed by Lina Persechini}

Thinking Machines: Art and Design in the Computer Age runs until 8 April 2018 at MoMA.

Published online: 6 April 2018

https://doi.org/10.1038/s41567-018-0110-3 